\title{
EFFECT OF DIFFERENT LEVELS OF NITROGEN AND MULCHING ON THE GROWTH OF CHINESE CABBAGE (Brassica campestris var. Pekinensis)
}

\author{
D. Easmin, M. J. Islam and K. Begum \\ Department of Horticulture and Postharvest Technology, Sher-e-Bangla \\ Agricultural University, Dhaka, Bangladesh
}

\begin{abstract}
The experiment was conducted in the Horticulture Farm of Sher-e-Bangla Agricultural University, Dhaka, Bangladesh during the period from October 2005 to December 2005 to study the effect of different levels of nitrogen and mulching on the growth and yield of Chinese cabbage. The tallest Chinese cabbage plant and spread of plant $(43.12 \mathrm{~cm}$ and $64.43 \mathrm{~cm})$ was recorded in $250 \mathrm{~kg}$ $\mathrm{N} / \mathrm{ha}$ and the shortest $(36.15 \mathrm{~cm}$ and $48.38 \mathrm{~cm})$ was recorded in $0 \mathrm{~kg} \mathrm{~N} / \mathrm{ha}$. The longest duration to start head formation (46.80 days) was recorded in $0 \mathrm{~kg} \mathrm{~N} / \mathrm{ha}$ and the shortest (40.96 days) was recorded in $250 \mathrm{~kg} \mathrm{~N} / \mathrm{ha}$. The highest marketable yield per hectare of Chinese cabbage (120.06 ton) was recorded in $250 \mathrm{~kg} \mathrm{~N} /$ ha and the lowest yield (67.90 ton) was recorded in $0 \mathrm{~kg} \mathrm{~N} / \mathrm{ha}$. The highest Chinese cabbage plant, spread of plant $(44.50 \mathrm{~cm}, 58.28 \mathrm{~cm})$ during harvest period was recorded in black polythene mulch and the shortest plant and spread of plant $(37.60 \mathrm{~cm}$ and $49.58 \mathrm{~cm})$ was recorded in no mulch i.e. control. The highest days to start of head formation of Chinese cabbage (45.37) was recorded in no mulch and the lowest days (41.17) were recorded in black polythene mulch. The highest marketable yield per hectare of Chinese cabbage (123.27 ton) was recorded in black polythene mulch and the lowest yield (76.51 ton) was recorded in no mulch. In each and every case maximum growth and yield contributing characters and yield was observed in $250 \mathrm{~kg} \mathrm{~N} / \mathrm{ha}$ and black polythene mulch and the reverse result was recorded in control condition i.e. no nitrogen and no mulch.
\end{abstract}

Key words : Mulching, Nitrogen, Chinese cabbage

\section{INTRODUCTION}

Chinese cabbage (Brassica campestris var. Pekinensis) is an important leafy, herbaceous vegetable crop originated in China and belongs to the family Cruciferae (Rashid, 1999). To attaining considerable production and quality yield for any crop it is necessary to proper management including ensuring the availability of essential nutrient components. Nitrogen has profound effect on the number of folded leaves and progressively increases the marketable yield (Obreza and Vavrina, 1993). A shortage of nitrogen during early growth may led to the condition known as "buttoning" when plant becomes stunted with reduce leaf development (Tindall, 1983). An adequate supply of nitrogen is essential for vegetative growth, head formation and desirable yield (Yoshizawa et al., 1981). On the 
other hand excessive application of nitrogen is not only uneconomical, but it can prolong the growing period and delay crop maturity. Excessive nitrogen application causes physiological disorder that appears as small black spots on the midribs of head leaves (Obreza and Vavrina, 1993). In Bangladesh, Chinese cabbage is grown during winter season where rainfall is scanty but it needs plenty of moisture of soil for its normal growth and development. Mulching play an important role to reduce the evaporation loss of soil and in this way, it maintain sufficient moisture in the soil. Mulching provide acceptable temperature to the soil by protecting sunlight. Mulching offers tremendous potential for increased crop production through its noticeable effect on the soil environment which ensures proper growth and yield of crop (Lal, 1989). The efficient use of nitrogen from the economic point of view can be achieved by soil moisture management through mulching. The efficiency of nitrogen fertilizer use was normally $30 \%$ under Bangladesh context, which was increased upto 53\% with special arrangement through mulching (Sweeney et al., 1987).

\section{MATERIALS AND METHODS}

The experiment was conducted in the Horticulture Farm of Sher-e-Bangla Agricultural University, Dhaka, Bangladesh during the period from October 2005 to December 2005 to study the effect of different levels of nitrogen and mulching on the growth and yield of Chinese cabbage. The experiment considered two factors. Factor A: Levels of nitrogen (4 levels) i.e. $\mathrm{N}_{0}$ (No $\mathrm{N}$ fertilizer/Control), $\mathrm{N}_{1}(160 \mathrm{~kg} / \mathrm{ha}), \mathrm{N}_{2}(200 \mathrm{~kg} / \mathrm{ha})$ and $\mathrm{N}_{3}(250$ $\mathrm{kg} / \mathrm{ha}$ ); Factor B: Mulches (4 levels) i.e. $\mathrm{M}_{0}$ (No mulch), $\mathrm{M}_{1}$ (black polythene), $\mathrm{M}_{2}$ (saw dust) and $\mathrm{M}_{3}$ (water hyacinth). The experiment was laid out in the two factors Randomized Complete Block Design (RCBD) with three replications. The significance of the difference among the treatment combinations means was estimated by the least significant difference (LSD) test at $5 \%$ level of probability (Gomez and Gomez, 1984).

\section{RESULTS AND DISCUSSION}

Increasing level of nitrogen fertilizer applied in the present experiment showed a gradual increase in plant height of Chinese cabbage starting from 20 DAS till harvest. The tallest plant $(43.12 \mathrm{~cm})$ during harvesting period was recorded in $250 \mathrm{~kg} \mathrm{~N} /$ ha which was statistically identical with $160 \mathrm{~kg} \mathrm{~N} /$ ha and $200 \mathrm{~kg} \mathrm{~N} /$ ha, respectively (42.25 and 41.76 $\mathrm{cm})$ and the shortest plant $(36.15 \mathrm{~cm})$ was recorded in the plot $0 \mathrm{~kg} \mathrm{~N} / \mathrm{ha}$. The results indicated that nitrogen fertilizer ensure the optimum condition for growth and development of plant. With the increase of nitrogen doses, plant height of Chinese cabbage increased up to certain level. All mulches used in the present experiment produced tallest plant of Chinese cabbage comparing with control (no mulch) starting from 20 DAS to harvest (Table 1). The tallest Chinese cabbage plant $(44.50 \mathrm{~cm})$ during harvesting period was recorded in black polythene mulch which was closely followed by saw dust mulch $(41.43 \mathrm{~cm})$. On the other hand, the shortest Chinese cabbage plant $(37.60$ $\mathrm{cm})$ was recorded in without mulch. Probably, mulches increase the moisture content of soil which also ensures the advanced growth of plant. Alam et al. (1989) reported similar trend of result. Combined effect of nitrogen and mulches demonstrated significant 
differences in consideration of plant height of Chinese cabbage. The maximum height of Chinese cabbage during harvest $(48.77 \mathrm{~cm})$ was observed in $250 \mathrm{~kg}$ N/ha and black polythene mulch and the shortest plant $(32.28 \mathrm{~cm})$ was recorded in control condition i.e. no nitrogen and no mulch (Table 2).

Table 1. Main effect of nitrogen and mulching on yield contributing characters of Chinese cabbage

\begin{tabular}{|c|c|c|c|c|c|c|c|c|}
\hline \multirow{2}{*}{$\begin{array}{l}\text { Nitrogen and } \\
\text { mulching }\end{array}$} & \multicolumn{4}{|c|}{ Plant height $(\mathrm{cm})$ at } & \multicolumn{4}{|c|}{ Spread of plant $(\mathrm{cm})$ at } \\
\hline & 20 DAS & 30 DAS & 40 DAS & Harvest & 20 DAS & 30 DAS & 40 DAS & Harvest \\
\hline $0 \mathrm{~kg} \mathrm{~N} / \mathrm{ha}\left(\mathrm{N}_{0}\right)$ & $15.08^{\mathrm{d}}$ & $26.76^{c}$ & $31.03^{\mathrm{b}}$ & $36.15^{b}$ & $22.37 \mathrm{c}$ & $42.81^{\mathrm{c}}$ & $44.99 \mathrm{~d}$ & $48.38^{\mathrm{d}}$ \\
\hline $160 \mathrm{~kg} N / \mathrm{ha}\left(\mathrm{N}_{1}\right)$ & $19.50 c$ & $32.27 \mathrm{~b}$ & $36.25^{a}$ & $42.25^{a}$ & $29.60^{b}$ & $49.87 \mathrm{~b}$ & $53.51^{c}$ & $57.23 c$ \\
\hline 200 kg N/ha $\left(N_{2}\right)$ & $20.96^{b}$ & $33.23^{\mathrm{ab}}$ & $36.02^{a}$ & $41.76^{\mathrm{a}}$ & $30.89 \mathrm{~b}$ & $51.96^{\mathrm{b}}$ & $57.82^{\mathrm{b}}$ & $61.52^{\mathrm{b}}$ \\
\hline 250 kg N/ha $\left(\mathrm{N}_{3}\right)$ & $22.65^{\mathrm{a}}$ & $34.45^{\mathrm{a}}$ & $37.11^{\mathrm{a}}$ & $43.12^{\mathrm{a}}$ & 32.57 a & $54.87 \mathrm{a}$ & $60.69 a$ & $64.43^{\mathrm{a}}$ \\
\hline LSD (0.05) & 1.342 & 1.755 & 1.594 & 1.545 & 1.512 & 2.108 & 1.927 & 2.228 \\
\hline Level of significance & 0.01 & 0.01 & 0.01 & 0.01 & 0.01 & 0.01 & 0.01 & 0.01 \\
\hline No mulch $\left(\mathrm{M}_{0}\right)$ & $16.82^{c}$ & $29.05^{c}$ & $32.25^{c}$ & $37.60^{d}$ & $25.23^{d}$ & $45.56^{c}$ & $49.58^{d}$ & $53.03^{d}$ \\
\hline Black polythene $\left(\mathrm{M}_{1}\right)$ & $22.17 \mathrm{a}$ & $33.95^{\mathrm{a}}$ & $38.17 \mathrm{a}$ & $44.50^{\mathrm{a}}$ & $31.92^{\mathrm{a}}$ & $53.25^{\mathrm{a}}$ & $58.28^{a}$ & $62.42^{a}$ \\
\hline Saw dust $\left(\mathrm{M}_{2}\right)$ & $20.24^{b}$ & $32.48^{\mathrm{ab}}$ & $35.70^{\mathrm{b}}$ & $41.43^{b}$ & $30.30^{b}$ & $51.64^{\mathrm{a}}$ & $56.01^{b}$ & $59.79 \mathrm{~b}$ \\
\hline Water hyacinth $\left(\mathrm{M}_{3}\right)$ & $18.96^{\mathrm{b}}$ & $31.23 \mathrm{~b}$ & $34.30^{\mathrm{b}}$ & $39.75^{c}$ & $27.99 c$ & $49.06^{\mathrm{b}}$ & $53.14 \mathrm{c}$ & $56.33^{c}$ \\
\hline LSD (0.05) & 1.342 & 1.755 & 1.594 & 1.545 & 1.512 & 2.108 & 1.927 & 2.228 \\
\hline Level of significance & 0.01 & 0.01 & 0.01 & 0.01 & 0.01 & 0.01 & 0.01 & 0.01 \\
\hline CV (\%) & 8.24 & 6.64 & 5.45 & 4.54 & 6.28 & 5.07 & 4.26 & 4.62 \\
\hline
\end{tabular}

All the level of nitrogen, which was applied in the present experiment showed a gradual increasing trend of plant spread of Chinese cabbage starting from 20 DAS to harvest. At harvest, the highest spread of Chinese cabbage plant $(64.43 \mathrm{~cm})$ was recorded in $250 \mathrm{~kg}$ $\mathrm{N} /$ ha which was closely followed by $200 \mathrm{~kg} \mathrm{~N} / \mathrm{ha}(61.52 \mathrm{~cm})$ and the lowest spread $(48.38$ $\mathrm{cm})$ was recorded in $0 \mathrm{~kg} \mathrm{~N} / \mathrm{ha}$ which was closely followed by $160 \mathrm{~kg} \mathrm{~N} / \mathrm{ha}$ $(57.23 \mathrm{~cm})$. Nitrogen fertilizer increases the growth and development of plant and the ultimate results is the highest spread of plant. Mulches used in the present research work showed increased spread of plant of Chinese cabbage comparing with control condition with no mulch starting from 20 DAS to harvest (Table 1). The highest spread of Chinese cabbage plant $(58.28 \mathrm{~cm})$ during harvesting was reported in black polythene mulch which was closely followed by saw dust mulch $(56.01 \mathrm{~cm})$. On the other hand the lowest spread of Chinese cabbage plant $(49.58 \mathrm{~cm})$ was recorded in no mulch which was closely followed by water hyacinth mulch $(53.14 \mathrm{~cm})$. Good spread of plant indicates favorable growth and development which was the ultimate result of mulches. Spread of plant showed significant combined effect due to nitrogen and mulches in Chinese cabbage. The maximum spread of Chinese cabbage plant at harvest $(69.84 \mathrm{~cm})$ was observed in $250 \mathrm{~kg}$ $\mathrm{N} /$ ha and black polythene mulch, while the minimum $(43.46 \mathrm{~cm})$ was recorded in control condition (Table 2). 
Table 2. Combined effect of nitrogen and mulching on yield contributing characters of Chinese cabbage

\begin{tabular}{|c|c|c|c|c|c|c|c|c|}
\hline \multirow{2}{*}{$\begin{array}{c}\text { Nitrogen } \times \\
\text { Mulching }\end{array}$} & \multicolumn{4}{|c|}{ Plant height $(\mathrm{cm})$ at } & \multicolumn{4}{|c|}{ Spread of plant $(\mathrm{cm})$ at } \\
\hline & 20 DAS & 30 DAS & 40 DAS & Harvest & 20 DAS & 30 DAS & 40 DAS & Harvest \\
\hline $\mathrm{N}_{0} \mathrm{M}_{0}$ & $11.37 \mathrm{~g}$ & $22.50^{\mathrm{f}}$ & $27.72 \mathrm{~g}$ & $32.28^{\mathrm{h}}$ & $17.26^{\mathrm{k}}$ & $39.07^{\mathrm{i}}$ & $40.24^{\mathrm{i}}$ & $43.46^{\mathrm{i}}$ \\
\hline $\mathrm{N}_{0} \mathrm{M}_{1}$ & $17.45^{\text {def }}$ & 29.50 cde & $32.26^{\mathrm{ef}}$ & $38.19^{f g}$ & $25.46^{h i j}$ & $45.53^{\mathrm{fgh}}$ & $48.72^{\mathrm{ij}}$ & $52.28 \mathrm{fg}$ \\
\hline $\mathrm{N}_{0} \mathrm{M}_{2}$ & $16.55^{\mathrm{ef}}$ & $28.46^{\mathrm{de}}$ & $30.63^{\mathrm{fg}}$ & $35.55 \mathrm{~g}$ & $24.32^{\mathrm{ij}}$ & $44.26 \mathrm{gh}$ & $47.33 \mathrm{jk}$ & $50.84 \mathrm{gh}$ \\
\hline $\mathrm{N}_{0} \mathrm{M}_{3}$ & $14.96^{\mathrm{f}}$ & $26.60 \mathrm{e}$ & 33.53 def & $38.56^{\mathrm{fg}}$ & $22.42 j$ & $42.40^{\mathrm{hi}}$ & $43.67 \mathrm{kl}$ & $46.96^{\mathrm{hi}}$ \\
\hline $\mathrm{N}_{1} \mathrm{M}_{0}$ & $18.34^{\mathrm{de}}$ & $30.48^{c d}$ & $33.61^{\text {def }}$ & $39.32^{\mathrm{ef}}$ & $28.03^{\mathrm{fgh}}$ & $46.33^{\text {efgh }}$ & $49.62^{\text {hij }}$ & $53.27 \mathrm{fg}$ \\
\hline $\mathrm{N}_{1} \mathrm{M}_{1}$ & $21.75^{\mathrm{bc}}$ & $33.35^{b c}$ & $38.80^{\mathrm{ab}}$ & $45.29 \mathrm{bc}$ & $31.32^{\text {cdef }}$ & $53.38 \mathrm{abc}$ & $57.91^{\text {cde }}$ & $61.72^{b c}$ \\
\hline $\mathrm{N}_{1} \mathrm{M}_{2}$ & 19.23 cde & $32.69 \mathrm{bc}$ & $36.26 \mathrm{bcd}$ & $42.16^{\text {cde }}$ & 30.37defg & $51.36 \mathrm{bcd}$ & $54.93^{\mathrm{efg}}$ & $58.90^{\text {cde }}$ \\
\hline $\mathrm{N}_{1} \mathrm{M}_{3}$ & $18.70^{\text {de }}$ & $32.57 \mathrm{bc}$ & $36.33^{\mathrm{bcd}}$ & 42.21 cde & $28.68^{\text {efgh }}$ & $48.40^{\operatorname{defg}}$ & 51.59ghi & $55.02^{\text {efg }}$ \\
\hline $\mathrm{N}_{2} \mathrm{M}_{0}$ & 18.60 de & $31.59 \mathrm{bcd}$ & $33.18^{\text {def }}$ & $38.82^{\mathrm{efg}}$ & $27.29 \mathrm{ghi}$ & 47.30 defg & $53.27 \mathrm{fgh}$ & $56.46^{\text {def }}$ \\
\hline $\mathrm{N}_{2} \mathrm{M}_{1}$ & $23.29 b$ & $35.57 \mathrm{ab}$ & $39.50 \mathrm{ab}$ & $45.76^{\mathrm{ab}}$ & $34.68 \mathrm{ab}$ & $56.34^{a}$ & $61.23 \mathrm{abc}$ & $65.84^{\mathrm{ab}}$ \\
\hline $\mathrm{N}_{2} \mathrm{M}_{2}$ & $21.69 \mathrm{bc}$ & $33.32^{\mathrm{bc}}$ & $37.34 \mathrm{bc}$ & $42.94 \mathrm{bcd}$ & $32.26 \mathrm{bcd}$ & $53.53^{\mathrm{abc}}$ & $59.34^{\mathrm{bcd}}$ & $62.90^{b c}$ \\
\hline $\mathrm{N}_{2} \mathrm{M}_{3}$ & $20.24 \mathrm{~cd}$ & $32.43^{b c}$ & $34.07 \mathrm{cdef}$ & $39.52^{\mathrm{ef}}$ & 29.33 defg & $50.66^{\text {bcde }}$ & $57.45^{\text {cdef }}$ & $60.89 \mathrm{~cd}$ \\
\hline $\mathrm{N}_{3} \mathrm{M}_{0}$ & $18.95^{\text {cde }}$ & $31.65^{\mathrm{bcd}}$ & $34.47 \mathrm{cde}$ & 39.97def & $28.33^{\text {efgh }}$ & $49.56^{\mathrm{cdef}}$ & $55.21^{\text {defg }}$ & $58.92^{\text {cde }}$ \\
\hline $\mathrm{N}_{3} \mathrm{M}_{1}$ & $26.21^{a}$ & $37.37 \mathrm{a}$ & $42.12^{\mathrm{a}}$ & $48.77 \mathrm{a}$ & $36.20^{a}$ & $57.75^{\mathrm{a}}$ & $65.25^{\mathrm{a}}$ & $69.84^{a}$ \\
\hline $\mathrm{N}_{3} \mathrm{M}_{2}$ & $23.50^{\mathrm{b}}$ & $35.45^{\mathrm{ab}}$ & $38.59^{b}$ & $45.04^{b c}$ & $34.24^{\mathrm{abc}}$ & $57.41^{\mathrm{a}}$ & $62.45^{\mathrm{ab}}$ & $66.53^{\mathrm{ab}}$ \\
\hline $\mathrm{N}_{3} \mathrm{M}_{3}$ & $21.96^{b c}$ & $33.33^{b c}$ & $33.27 \mathrm{def}$ & $38.70^{\mathrm{efg}}$ & $31.52^{\text {bcde }}$ & $54.76^{\mathrm{ab}}$ & $59.86^{\mathrm{bc}}$ & $62.45^{\mathrm{bc}}$ \\
\hline LSD (0.05) & 2.685 & 3.510 & 3.189 & 3.089 & 3.024 & 4.215 & 3.855 & 4.456 \\
\hline $\begin{array}{l}\text { Level of } \\
\text { significance }\end{array}$ & 0.01 & 0.01 & 0.05 & 0.01 & 0.05 & 0.01 & 0.01 & 0.01 \\
\hline CV $(\%)$ & 8.24 & 6.64 & 5.45 & 4.54 & 6.28 & 5.07 & 4.26 & 4.62 \\
\hline
\end{tabular}

Root length of Chinese cabbage showed statistically significant variation and longest roots per plant of Chinese cabbage $(26.88 \mathrm{~cm})$ was recorded in $250 \mathrm{~kg} \mathrm{~N} /$ ha which was statistically similar to $200 \mathrm{~kg} \mathrm{~N} /$ ha and $160 \mathrm{~kg} \mathrm{~N} / \mathrm{ha}(26.23 \mathrm{~cm}$ and $25.11 \mathrm{~cm}$, respectively). The lowest length of roots per plant of Chinese cabbage $(20.37 \mathrm{~cm})$ was recorded from 0 $\mathrm{kg} \mathrm{N} / \mathrm{ha}$ (Table 3). The longest root per plant of Chinese cabbage $(26.92 \mathrm{~cm})$ was recorded in black polythene mulch which was statistically parallel by saw dust mulch $(25.27 \mathrm{~cm})$. On the other hand, the shortest root per plant of Chinese cabbage $(22.16 \mathrm{~cm})$ was recorded in no mulch which was closely followed to water hyacinth mulch $(24.24 \mathrm{~cm})$. Generally, mulches ensure the optimum soil moisture for proper root development, and these conditions enhance root development as well as increase root length (Table 3). In consideration the combined effect of nitrogen and mulches length of roots per plant in Chinese cabbage showed statistically distinction. The highest length of roots per plant $(29.42 \mathrm{~cm})$ was recorded in $250 \mathrm{~kg} \mathrm{~N} / \mathrm{ha}$ and black polythene mulch and the lowest $(15.72 \mathrm{~cm})$ was recorded in control condition (Table 4$)$. 
Table 3. Main effect of nitrogen and mulching on yield contributing characters and yield of Chinese cabbage

\begin{tabular}{l|c|c|c|c|c}
\hline $\begin{array}{c}\text { Nitrogen and } \\
\text { mulching }\end{array}$ & $\begin{array}{c}\text { Root length } \\
(\mathrm{cm})\end{array}$ & $\begin{array}{c}\text { Folded } \\
\text { leaves/plant } \\
(\text { No. })\end{array}$ & $\begin{array}{c}\text { Unfolded } \\
\text { leaves/plant } \\
(\text { No. })\end{array}$ & $\begin{array}{c}\text { Days to } \\
\text { initiation of } \\
\text { head }\end{array}$ & $\begin{array}{c}\text { Marketable } \\
\text { yield (t/ha) }\end{array}$ \\
\hline $0 \mathrm{~kg} \mathrm{~N} /$ ha $\left(\mathrm{N}_{0}\right)$ & $20.37^{\mathrm{b}}$ & $42.96^{\mathrm{c}}$ & $15.25^{\mathrm{a}}$ & $46.80^{\mathrm{a}}$ & $67.90^{\mathrm{c}}$ \\
$160 \mathrm{~kg} \mathrm{~N} / \mathrm{ha}\left(\mathrm{N}_{1}\right)$ & $25.11^{\mathrm{a}}$ & $51.95^{\mathrm{b}}$ & $13.35^{\mathrm{b}}$ & $41.37^{\mathrm{b}}$ & $107.09^{\mathrm{b}}$ \\
$200 \mathrm{~kg} \mathrm{~N} /$ ha $\left(\mathrm{N}_{2}\right)$ & $26.23^{\mathrm{a}}$ & $52.86^{\mathrm{b}}$ & $12.91^{\mathrm{b}}$ & $42.36^{\mathrm{b}}$ & $110.65^{\mathrm{b}}$ \\
$250 \mathrm{~kg} \mathrm{~N} /$ ha $\left(\mathrm{N}_{3}\right)$ & $26.88^{\mathrm{a}}$ & $55.51^{\mathrm{a}}$ & $11.68^{\mathrm{c}}$ & $40.96^{\mathrm{b}}$ & $120.06^{\mathrm{a}}$ \\
\hline LSD (0.05) & 1.724 & 2.009 & 0.838 & 1.730 & 5.342 \\
\hline Level of significance & 0.01 & 0.01 & 0.01 & 0.01 & 0.01 \\
\hline No mulch $\left(\mathrm{M}_{0}\right)$ & $22.16^{\mathrm{c}}$ & $46.82^{\mathrm{d}}$ & $14.91^{\mathrm{a}}$ & $45.37^{\mathrm{a}}$ & $76.51^{\mathrm{d}}$ \\
Black polythene $\left(\mathrm{M}_{1}\right)$ & $26.92^{\mathrm{a}}$ & $54.47^{\mathrm{a}}$ & $12.07^{\mathrm{c}}$ & $41.17^{\mathrm{c}}$ & $123.27^{\mathrm{a}}$ \\
Saw dust $\left(\mathrm{M}_{2}\right)$ & $25.27^{\mathrm{ab}}$ & $52.13^{\mathrm{b}}$ & $12.83^{\mathrm{bc}}$ & $41.67^{\mathrm{bc}}$ & $111.01^{\mathrm{b}}$ \\
Water hyacinth $\left(\mathrm{M}_{3}\right)$ & $24.24^{\mathrm{b}}$ & $49.86^{\mathrm{c}}$ & $13.39 \mathrm{~b}$ & $43.27^{\mathrm{b}}$ & $94.91^{\mathrm{c}}$ \\
\hline LSD (0.05) & 1.724 & 2.009 & 0.838 & 1.730 & 5.342 \\
\hline Level of significance & 0.01 & 0.01 & 0.01 & 0.01 & 0.01 \\
\hline CV (\%) & 8.39 & 4.74 & 7.56 & 4.84 & 6.32 \\
\hline
\end{tabular}

All the levels of nitrogen fertilizer showed a steady increasing trend in the number of folded leaves. The highest number of folded leaves of Chinese cabbage (55.51) was recorded in $250 \mathrm{~kg} \mathrm{~N} / \mathrm{ha}$. Application of $200 \mathrm{~kg} \mathrm{~N} / \mathrm{ha}$ and $160 \mathrm{~kg} \mathrm{~N} /$ ha showed statistically similar results in this experiment. The lowest number of folded leaves for Chinese cabbage (42.96) was reported in $0 \mathrm{~kg} \mathrm{~N} / \mathrm{ha}$. Mulches that were used in the present trial showed increase number of folded leaves of Chinese cabbage considering with control. The maximum number of folded leaves for Chinese cabbage plant (54.47) during harvest was recorded in black polythene mulch which was statistically identical with saw dust mulch (52.13). On the other hand the minimum number of folded leaves of Chinese cabbage plant (46.82) was recorded in no mulch which was statistically similar with water hyacinth mulch (49.86). Hill (1982) recorded the highest folded leaves from black polythene mulch. Combined effect of nitrogen and mulches showed a statistically significant difference in consideration of number of folded leaves/plant. Maximum number of folded leaves (58.66) of Chinese cabbage at harvest was observed in $250 \mathrm{~kg}$ $\mathrm{N} /$ ha and black polythene mulch, where the minimum number of folded leaves/plant (32.81) was recorded in control condition (Table 4).

Nitrogen fertilizer showed a gradual decreasing propensity of number of unfolded leaves per plant of Chinese cabbage. The highest number of unfolded leaves per plant of Chinese cabbage (15.23) was recorded in $0 \mathrm{~kg} \mathrm{~N} /$ ha while the lowest number (11.68) was recorded from $250 \mathrm{~kg} \mathrm{~N} /$ ha which was closely followed by $200 \mathrm{~kg} \mathrm{~N} /$ ha (12.91). Number of unfolded leaves/plant at different mulches showed a statistically significant variation. 
The highest number of unfolded leaves per plant of Chinese cabbage (14.91) was recorded in no mulch which was closely followed by water hyacinth mulch (13.39). On the other hand the lowest number (12.07) was recorded in black polythene mulch which was statistically similar with saw dust mulch (12.83). Number of unfolded leaves per plant in Chinese cabbage showed statistically significant difference in relation with combined effect of nitrogen and mulches. The maximum number of unfolded leaves per plant (16.60) was recorded in control, while the minimum (10.13) was recorded in $250 \mathrm{~kg}$ $\mathrm{N} /$ ha and black polythene mulch.

Table 4. Combined effect of nitrogen and mulching on yield contributing characters and yield of Chinese cabbage

\begin{tabular}{|c|c|c|c|c|c|}
\hline $\begin{array}{c}\text { Nitrogen } \times \\
\text { Mulching }\end{array}$ & $\begin{array}{l}\text { Root length } \\
\quad(\mathrm{cm})\end{array}$ & $\begin{array}{c}\text { Folded } \\
\text { leaves/plant } \\
(\text { No.) }\end{array}$ & $\begin{array}{c}\text { Unfolded } \\
\text { leaves/plant } \\
\text { (No.) }\end{array}$ & $\begin{array}{c}\text { Days to } \\
\text { initiation of } \\
\text { head }\end{array}$ & $\begin{array}{l}\text { Marketable } \\
\text { yield (t/ha) }\end{array}$ \\
\hline $\mathrm{N}_{0} \mathrm{M}_{0}$ & $15.72^{\mathrm{f}}$ & $32.81^{\mathrm{i}}$ & $16.60^{a}$ & $48.51^{a}$ & $45.60^{\mathrm{i}}$ \\
\hline $\mathrm{N}_{0} \mathrm{M}_{1}$ & 23.22cde & $48.54 \mathrm{fg}$ & $13.75^{\mathrm{cd}}$ & $46.59 \mathrm{ab}$ & $81.21 \mathrm{fg}$ \\
\hline $\mathrm{N}_{0} \mathrm{M}_{2}$ & $21.65^{\text {de }}$ & $46.86^{\mathrm{gh}}$ & $14.24^{\mathrm{cd}}$ & $45.57 \mathrm{abc}$ & $76.23 \mathrm{gh}$ \\
\hline $\mathrm{N}_{0} \mathrm{M}_{3}$ & $20.90 \mathrm{e}$ & $43.61^{\mathrm{h}}$ & $16.41^{\mathrm{ab}}$ & $46.54^{\mathrm{ab}}$ & $68.56^{\mathrm{h}}$ \\
\hline $\mathrm{N}_{1} \mathrm{M}_{0}$ & 23.63 cde & $49.76^{\text {defg }}$ & $14.77 \mathrm{bc}$ & $45.48^{\mathrm{abc}}$ & $81.62^{\mathrm{fg}}$ \\
\hline $\mathrm{N}_{1} \mathrm{M}_{1}$ & $26.93^{a b c}$ & $54.25^{\mathrm{abcd}}$ & $12.63 \mathrm{def}$ & $38.81 \mathrm{~g}$ & $131.80^{\mathrm{b}}$ \\
\hline $\mathrm{N}_{1} \mathrm{M}_{2}$ & $25.23 \mathrm{bcd}$ & $52.50^{\text {bcdef }}$ & $13.12^{\text {cdef }}$ & $40.49^{\mathrm{efg}}$ & $116.11^{c}$ \\
\hline $\mathrm{N}_{1} \mathrm{M}_{3}$ & 24.65 bcde & 51.31 cdefg & $12.91^{\text {cdef }}$ & $40.68^{\mathrm{efg}}$ & $98.83 e$ \\
\hline $\mathrm{N}_{2} \mathrm{M}_{0}$ & $24.75^{\text {bcde }}$ & $50.78^{c d e f g}$ & $14.71^{b c}$ & $44.67 \mathrm{abcd}$ & $86.47 \mathrm{fg}$ \\
\hline $\mathrm{N}_{2} \mathrm{M}_{1}$ & $28.12^{\mathrm{ab}}$ & $56.44^{\mathrm{ab}}$ & 11.75 efg & 39.93efg & $135.41^{\mathrm{ab}}$ \\
\hline $\mathrm{N}_{2} \mathrm{M}_{2}$ & $26.40^{a b c}$ & $54.60^{\mathrm{abc}}$ & $12.52^{\mathrm{def}}$ & $41.34^{\mathrm{defg}}$ & $118.90 c$ \\
\hline $\mathrm{N}_{2} \mathrm{M}_{3}$ & $25.67 \mathrm{abc}$ & $49.63^{\mathrm{efg}}$ & 12.68 def & $43.51^{\text {bcde }}$ & $101.82^{d e}$ \\
\hline $\mathrm{N}_{3} \mathrm{M}_{0}$ & 24.53 bcde & 53.93 bcde & 13.57 cde & $42.84^{\text {bcdef }}$ & $92.36^{\mathrm{ef}}$ \\
\hline $\mathrm{N}_{3} \mathrm{M}_{1}$ & $29.42^{\mathrm{a}}$ & $58.66^{a}$ & $10.13 \mathrm{~g}$ & $39.35 \mathrm{fg}$ & $144.67^{\mathrm{a}}$ \\
\hline $\mathrm{N}_{3} \mathrm{M}_{2}$ & $27.80^{\mathrm{ab}}$ & $54.55^{\mathrm{abc}}$ & $11.45^{\mathrm{fg}}$ & $39.29 \mathrm{fg}$ & $132.78^{b}$ \\
\hline $\mathrm{N}_{3} \mathrm{M}_{3}$ & $25.75^{\mathrm{abc}}$ & $54.88^{\mathrm{abc}}$ & $11.58^{\mathrm{fg}}$ & 42.37cdefg & $110.44^{\mathrm{cd}}$ \\
\hline LSD (0.05) & 3.448 & 4.018 & 1.677 & 3.460 & 10.68 \\
\hline $\begin{array}{l}\text { Level of } \\
\text { significance }\end{array}$ & 0.05 & 0.01 & 0.01 & 0.01 & 0.01 \\
\hline $\mathrm{CV}(\%)$ & 8.39 & 4.74 & 7.56 & 4.84 & 6.32 \\
\hline
\end{tabular}

Days to start of head formation from seed sowing of Chinese cabbage showed significant differences with different levels of nitrogen fertilizer application in the present trial. All the level of nitrogen fertilizer showed a gradual decreasing tendency of days to starting head formation of Chinese cabbage (Table 3). The longest duration to starting head formation (46.80) was recorded in $0 \mathrm{~kg} \mathrm{~N} / \mathrm{ha}$. On the other hand, the shortest duration to starting head formation (40.96) was recorded in the plot with $250 \mathrm{~kg} \mathrm{~N} / \mathrm{ha}$. Days to start 
of head formation at different mulches showed differences. The longest duration to starting of head formation of Chinese cabbage (45.37) was recorded in no mulch which was closely followed by water hyacinth mulch (43.27). On the other hand the shortest duration (41.17) was recorded in black polythene mulch. Days to start of head formation in Chinese cabbage showed statistical difference in relation with combined effect of nitrogen and mulches. The shortest duration (39.29) was recorded in $250 \mathrm{~kg} \mathrm{~N} / \mathrm{ha}$ and saw dust mulch, where the longest (48.51) was recorded in control (Table 4).

Marketable yield per hectare showed statistically significant differences with different doses of nitrogen fertilizer applied in the present experiment. Nitrogen fertilizer showed a gradual increasing tendency to marketable yield per hectare. The highest marketable yield per hectare (120.06 ton) was recorded in $250 \mathrm{~kg} \mathrm{~N} /$ ha which was closely followed by $200 \mathrm{~kg} \mathrm{~N} /$ ha and $160 \mathrm{~kg} \mathrm{~N} / \mathrm{ha}$ (110.65 and 107.09 ton). On another way the lowest marketable yield per hectare of Chinese cabbage (67.90 ton) was recorded in control. Marketable yield per hectare at different mulches showed significant difference. The highest marketable yield per hectare (123.27 ton) was recorded in black polythene mulch which was closely followed by saw dust mulch (111.01 ton) and the lowest marketable yield (76.51 ton) was recorded in no mulch which was closely followed by water hyacinth mulch (94.91 ton). Marketable yield per hectare showed statistically significant variation in relation with combined effect of nitrogen and mulches. The maximum marketable yield per hectare (144.67 ton) was recorded in $250 \mathrm{~kg} \mathrm{~N} /$ ha and black polythene mulch, while the minimum (45.60 ton) was recorded in control condition i.e. no nitrogen no mulch.

\section{REFERENCES}

Alam, M. S., Iqbal, T. M. T., Amin, M. and Gaffar, M. A. 1989. Krishitattic Fasaler Utpadan O Unnayan (in Bengali). T. M. Jubair Bin Iqbal, Sirajgonj. pp. 231-239.

Gomez, K. A. and Gomez, A. A. 1984. Statistical Procedure for Agricultural Research (2nd edn.). Int. Rice Res. Inst., A Willey Int. Sci., Pub., pp. 28-192.

Hill, D. E., Hankin, L. and Stephens, G. R. 1982. Mulches: their effect on fruit timing and yields of vegetables. Bull. Con. Agric. Expt. Sta., New Haven.

Lal M. H. 1989. Effect of mulching on Chinese cabbage. Acta Agriculturae Scandinavica Soil and Plant Science. 12(1): 12-13.

Obreza, T. A. and Vavrina, C. S. 1993. Production of Chinese cabbage in relation to nitrogen source. Rate and Leaf Nutrient Concentration in Soil Science and Plant Analysis. 24: 13-14 [Cited from Hort. Abstr., 1994, 64(4): 2751].

Rashid, M. M. 1999. Sabjii Biggan. Rashid Publishing. House 94. Old DOHS. Dhaka-1206. p. 248.

Sweeney, D. W., Graetz, D. A., Bottehers, A. B., Locasio, S. J. and Campbel, K. L. 1987. Tomato yield and nitrogen recovery as influence by irrigation method, nitrogen source and mulch. Hort. Sci., 22(1): 27-29.

Tindall, H. D. 1983. Vegetable in the Tropics. MacMillan Education Ltd. Houndmills. London [Cited from Hort. Abstr., 50(6): 3791-1984].

Yoshizawa, T. C. H. M. and Roan, Y. C. 1981. Management of summer Chinese cabbage in Taiwan Chinese cabbage. AVRDC, Shanhua, Taiwan. p. 125. 